APRIL, 1973

\title{
SERIES EQUATIONS INVOLVING JACOBI POLYNOMIALS AND MIXED BOUNDARY-VALUE PROBLEMS OF THE LAPLACE EQUATION*
}

\author{
BY
}

\author{
MASAAKI SHIMASAKI AND TAKESHI KIYONO
}

Kyoto University

\begin{abstract}
A formal analy'sis of series equations involving Jacobi polynomials is given. $(2 N+1)$ series equations involving Jacobi polynomials are reduced to a set of $N$ simultaneous Fredholm integral equations which can be solved numerically by the use of the Legendre-Gauss quadrature formula. In case of triple series equations the result is in agreement with that of Lowndes. Besides triple series equations, certain quadruple series equations can be also reduced to a single Fredholm integral equation of the second kind. Owing to the introduction of an arbitrary weight factor, the theory is feasible for the analysis of various many-part mixed boundary-value problems of the Laplace equation. As an example, special cases of certain trigonometric series equations are discussed in detail in connection with an electrostatic problem.

1. Introduction. The theory of "dual integral equations" or "dual series equations" has made remarkable progress in connection with mixed boundary-value problems in potential theory in these two decades [6]. As far as series equations involving Jacobi polynomials are concerned, dual series equations were studied by Noble [5] in 1963. Srivastav [7] gave the solution of a different kind of dual series equations. In 1968 Lowndes [4] studied triple series equations as a generalization of Noble's dual series equations. His result shows that triple series equations can be reduced to a Fredholm integral equation of the second kind and that Srivastava's result on triple series equations [8] is reduced to its special case.

It is important to study multiple pairs of series equations not only for the theory of series equations itself but for the application of the theory to boundary-value problems. In this paper a formal analysis of certain $(2 N+1)$ series equations involving Jacobi polynomials is given. The series equations are reduced to a set of $N$ simultaneous Fredholm integral equations. It is interesting to investigate the case in which the problem can be reduced to a single integral equation. Besides triple series equations studied by Lowndes [4], certain quadruple series equations can be also reduced to a single integral equation. Although application of the theory of dual series equations or triple series equations is restricted to the analysis of two-part or three-part mixed boundary-value problems, we can now analyze many-part mixed boundary-value problems by the method presented in this paper.

Our analysis is purely formal and no justification is given for various limiting processes such as change of the order of integrations.
\end{abstract}

* Received December 14, 1971. 
2. $(2 N+1)$ series equations involving Jacobi polynomials. Let $0=\rho_{0} \leqq \rho_{1}<$ $\rho_{2}<\cdots<\rho_{2 N} \leqq \rho_{2 N+1}=1$. Consider the following $(2 N+1)$ series equations involving Jacobi polynomial:

$$
\begin{array}{r}
\sum_{n=0}^{\infty} C_{n} J_{n}(a, \lambda ; \rho)=0, \rho_{2(i-1)}<\rho<\rho_{2 i-1}, \quad i=1,2, \cdots, N+1, \\
\sum_{n=0}^{\infty} C_{n} p_{n}(\lambda-\sigma, \lambda)\left(1+\tilde{H}_{n}\right) J_{n}(a, \lambda ; \rho)=g_{2 i}(\rho), \rho_{2 i-1}<\rho<\rho_{2 i}, \\
i=1,2, \cdots, N,
\end{array}
$$

where $\tilde{H}_{n}$ 's and $g_{2 i}(\rho)$ 's are known series and known functions, respectively,

$$
p_{n}(\lambda-\sigma, \lambda)=\frac{\Gamma(\lambda-\sigma+n) \Gamma(1+a-\lambda+n)}{\Gamma(\lambda+n) \Gamma(1+a+\sigma-\lambda+n)},
$$

and $J_{n}(a, \lambda ; \rho)$ denotes the Jacobi polynomial $J_{n}(a, \lambda ; \rho)={ }_{2} F_{1}(a+n,-n ; \lambda ; \rho)$. We assume that $a+1>\lambda>\sigma, 0<\sigma<1$. We further assume that the arbitrary weight factor $\tilde{H}_{n}$ has a sufficiently rapid convergence property. The introduction of the factor $\widetilde{H}_{n}$ substantially extends the applicability of the theory to mixed boundary-value problems.

3. Reduction to simultaneous Fredholm integral equations. In this section we show that series equations (2.1) and (2.2) can be reduced to simultaneous Fredholm integral equations of the second kind which can be solved by a standard numerical method.

The orthogonality relations for the Jacobi polynomials can be written in the form

$$
\int_{0}^{1} r^{\lambda-1}(1-r)^{a-\lambda} J_{m}(a, \lambda ; r) J_{n}(a, \lambda ; r) d r=\frac{\delta_{m n}}{\triangle_{n}^{2}}, \quad a+1>\lambda>0,
$$

where $\delta_{m n}$ is the Kronecker symbol and

$$
\triangle_{n}^{2}=\frac{(a+2 n) \Gamma(a+n) \Gamma(\lambda+n)}{\Gamma(n+1)\{\Gamma(\lambda)\}^{2} \Gamma(1+a-\lambda+n)} .
$$

We write

$$
\sum_{n=0}^{\infty} C_{n} J_{n}(a, \lambda ; \rho)=(1-\rho)^{\lambda-a} \psi_{2 i}(\rho), \rho_{2 i-1}<\rho<\rho_{2 i}, \quad i=1,2, \cdots, N,
$$

where the $\psi_{2 i}(\rho)$ 's $(1 \leqq i \leqq N)$ are unknown functions. From (2.1), (3.1) and (3.3), we obtain

$$
C_{n}=\triangle_{n}^{2} \sum_{j=1}^{N} \int_{\rho_{3 j-1}}^{\rho_{3 i}} \psi_{2 j}(r) r^{\lambda-1} J_{n}(a, \lambda ; r) d r
$$

If we substitute (3.4) into (2.2), then series equations (2.1) and (2.2) can be transformed into integral equations. We further define new functions $\Psi_{2 i}(x)$ by

$$
\Psi_{2 i}(x)=\int_{x}^{\rho_{1 i}} \frac{\psi_{2 i}(r)}{(r-x)^{1-\sigma}} d r, \rho_{2 i-1}<x<\rho_{2 i}, \quad i=1,2, \cdots, N .
$$

From the theory of the Volterra integral equation of the first kind (e.g. [6]), the function $\psi_{2 i}(r)$ can be expressed as 


$$
\psi_{2 i}(r)=-\frac{\sin \sigma \pi}{\pi} \frac{d}{d r} \int_{r}^{\rho: i} \frac{\Psi_{2 i}(y)}{(y-r)^{\sigma}} d y .
$$

In this section we derive simultaneous Fredholm integral equations of $\Psi_{2 i}(x)$. Once these integral equations are solved, $\psi_{2 i}(r)$ and $C_{n}$ can be determined by (3.6) and (3.4), respectively. The following lemmas play an important role in the derivation of the integral equations.

Lemma. 1. The following relation is due to Noble [5]:

$$
\begin{aligned}
k(r, \rho) & =\{\Gamma(\sigma)\}^{2}(r \rho)^{\lambda-1} \sum_{n=0}^{\infty} \triangle_{n}^{2} p_{n}(\lambda-\sigma, \lambda) J_{n}(a, \lambda ; r) J_{n}(a, \lambda ; \rho) \\
& =\int_{0}^{t} m(x)(r-x)^{\sigma-1}(\rho-x)^{\sigma-1} d x \\
& =k_{t}(r, \rho),
\end{aligned}
$$

where

$$
m(x)=x^{\lambda-\sigma-1}(1-x)^{\lambda-\sigma-a}, \quad t=\min (r, \rho) .
$$

2. The following definite integral involving $\psi_{2 i}(r)$ can be transformed into that involving $\Psi_{2 i}(x)$ as

$$
\int_{\rho_{2 i-1}}^{\rho_{2 i}} \frac{\psi_{2 i}(r)}{(r-x)^{1-\sigma}} d r=\int_{\rho_{s i-1}}^{\rho_{2 i}} T\left(\sigma, \rho_{2 i-1} ; x, y\right) \Psi_{2 i}(y) d y, x<\rho_{2 i-1},
$$

where $T(\sigma, u ; x, y)$ is defined by

$$
T(\sigma, u ; x, y)=\frac{\sin \sigma \pi}{\pi} \frac{(u-x)^{\sigma}}{(y-x)(y-u)^{\sigma}}, \quad x<u<y .
$$

3. The function $T(\sigma, u ; x, y)$ defined by (3.10) satisfies

$$
\frac{\sin \sigma \pi}{\pi} \frac{d}{d x} \int_{u}^{x} \frac{d \rho}{(x-\rho)^{\sigma}(\rho-y)^{1-\sigma}}=T(\sigma, u ; y, x) .
$$

4. We define a function $I_{n}(a, \lambda, \sigma, d ; x)$ by

$$
I_{n}(a, \lambda, \sigma, d ; x)=\frac{d}{d x} \int_{d}^{x} \frac{\rho^{\lambda-1}}{(x-\rho)^{\sigma}} J_{n}(a, \lambda ; \rho) d \rho .
$$

Then we have the following relation:

$$
\begin{aligned}
\frac{\Gamma(\sigma)}{\Gamma(1-\sigma)} \frac{d}{d x} \int_{d}^{x} & \frac{\rho^{\lambda-1}}{(x-\rho)^{\sigma}} d \rho \sum_{n=0}^{\infty} \tilde{H}_{n} \triangle_{n}^{2} p_{n}(\lambda-\sigma, \lambda) \int_{\rho_{\mathbf{3} j-1}}^{\rho_{\mathbf{s} j}} \psi_{2 j}(r) r^{\lambda-1} J_{n}(a, \lambda ; \rho) J_{n}(a, \lambda ; r) d r \\
= & \int_{\rho_{\mathbf{s i - 1}}}^{\rho_{\mathbf{s} j}} \Psi_{2 j}(y) d y\left\{\frac{\sin \sigma \pi}{\pi} \frac{\Gamma(\sigma)}{\Gamma(1-\sigma)}\right. \\
& \left.\cdot \sum_{n=0}^{\infty} \tilde{H}_{n} \triangle_{n}^{2} p_{n}(\lambda-\sigma, \lambda) I_{n}(a, \lambda, \sigma, d ; x) I_{n}\left(a, \lambda, \sigma, \rho_{2 j-1} ; y\right)\right\} .
\end{aligned}
$$

Proof. 2: derivation of (3.9). Substitution of (3.6) into the left-hand side of (3.9) and the use of integration by parts give 


$$
\begin{aligned}
& \int_{\rho 3 i-1}^{\rho_{3 i}} \frac{\psi_{2 i}(r)}{(r-x)^{1-\sigma}} d r=-\frac{\sin \sigma \pi}{\pi} \int_{\rho_{3 i-1}}^{\rho_{3 i}} \frac{1}{(r-x)^{1-\sigma}}\left\{\frac{d}{d r} \int_{r}^{\rho_{2 i}} \frac{\Psi_{2 i}(y)}{(y-r)^{\sigma}} d y\right\} d r \\
& =-\frac{\sin \sigma \pi}{\pi}\left[\frac{1}{(r-x)^{1-\sigma}} \int_{r}^{\rho, i} \frac{\Psi_{2 i}(y)}{(y-r)^{\sigma}} d y\right]_{r=\rho_{2 i-1}}^{r=\rho_{2 i}} \\
& -\frac{\sin \sigma \pi}{\pi} \int_{\rho_{\mathbf{s i}-1}}^{\rho_{\mathbf{s i}}} \frac{1-\sigma}{(r-x)^{2-\sigma}} d r \int_{r}^{\rho_{\mathbf{S i}}} \frac{\Psi_{2 i}(y)}{(y-r)^{\sigma}} d y=\frac{\sin \sigma \pi}{\pi} \int_{\rho_{\mathbf{s i}-\mathbf{i}}}^{\rho_{\mathbf{s i}}} \Psi_{2 i}(y) d y \\
& \text {. }\left\{\frac{1}{\left(\rho_{2 i-1}-x\right)^{1-\sigma}\left(y-\rho_{2 i-1}\right)^{\sigma}}-(1-\sigma) \int_{\rho_{3 i-1}}^{\nu} \frac{d r}{(r-x)^{2-\sigma}(y-r)^{\sigma}}\right\} \text {. }
\end{aligned}
$$

Since

$$
(1-\sigma) \int_{\rho_{i-i-1}}^{\nu} \frac{d r}{(r-x)^{2-\sigma}(y-r)^{\sigma}}=\frac{1}{y-x}\left(\frac{y-\rho_{2 i-1}}{\rho_{2 i-1}-x}\right)^{1-\sigma}
$$

we have

$$
\begin{aligned}
\int_{\rho_{3 i-1}}^{\rho_{3 i}} \frac{\psi_{2 i}(r)}{(r-x)^{1-\sigma}} d r & =\frac{\sin \sigma \pi}{\pi} \int_{\rho_{3 i-1}}^{\rho_{2 i}} \frac{\left(\rho_{2 i-1}-x\right)^{\sigma}}{(y-x)\left(y-\rho_{2 i-1}\right)^{\sigma}} \Psi_{2 i}(y) d y \\
& =\int_{\rho_{3 i-1}}^{\rho_{3 i}} T\left(\sigma, \rho_{2 i-1} ; x, y\right) \Psi_{2 i}(y) d y .
\end{aligned}
$$

3: derivation of (3.11). With the aid of integration by parts, we obtain

$$
\int_{u}^{x} \frac{d \rho}{(x-\rho)^{\sigma}(\rho-y)^{1-\sigma}}=\frac{1}{1-\sigma}\left(\frac{x-u}{u-y}\right)^{1-\sigma}-\int_{u}^{x} \frac{(x-\rho)^{1-\sigma}}{(\rho-y)^{2-\sigma}} d \rho .
$$

Differentiation of the above equation with respect to $x$ gives $\frac{\sin \sigma \pi}{\pi} \frac{d}{d x} \int_{u}^{x} \frac{d \rho}{(x-\rho)^{\sigma}(\rho-y)^{1-\sigma}}$

$$
\begin{aligned}
& =\frac{\sin \sigma \pi}{\pi}\left\{\frac{1}{(x-u)^{\sigma}(u-y)^{1-\sigma}}-(1-\sigma) \int_{u}^{x} \frac{d \rho}{(x-\rho)^{\sigma}(\rho-y)^{2-\sigma}}\right\} \\
& =\frac{\sin \sigma \pi}{\pi} \frac{(u-y)^{\sigma}}{(x-y)(x-u)^{\sigma}} \\
& =T(\sigma, u ; y, x) .
\end{aligned}
$$

4: derivation of (3.13). From (3.12), one can see that the function $I_{n}\left(a, \lambda, \sigma, \rho_{2,-1} ; x\right)$ satisfies the integral equation

$$
r^{\lambda-1} J_{n}(a, \lambda ; r)=\frac{\sin \sigma \pi}{\pi} \int_{\rho, i-1}^{r} \frac{I_{n}\left(a, \lambda, \sigma, \rho_{2 i-1} ; y\right)}{(r-y)^{1-\sigma}} d y .
$$

From (3.5) and (3.14), we have for the left-hand side of (3.13),

$$
\begin{aligned}
& \frac{\Gamma(\sigma)}{\Gamma(1-\sigma)} \sum_{n=0}^{\infty} \tilde{H}_{n} \triangle_{n}^{2} p_{n}(\lambda-\sigma, \lambda) I_{n}(a, \lambda, \sigma, d ; x) \int_{\rho_{3 i-1}}^{\rho, j} \psi_{2 j}(r) d r \frac{\sin \sigma \pi}{\pi} \\
& \cdot \int_{\rho, 1-1}^{r} \frac{I_{n}\left(a, \lambda, \sigma, \rho_{2 j-1} ; y\right)}{(r-y)^{1-\sigma}} d y=\frac{\sin \sigma \pi}{\pi} \frac{\Gamma(\sigma)}{\Gamma(1-\sigma)} \sum_{n=0}^{\infty} \tilde{H}_{n} \triangle_{n}^{2} p_{n}(\lambda-\sigma, \lambda) I_{n}(a, \lambda, \sigma, d ; x)
\end{aligned}
$$


$\cdot \int_{\rho_{\mathbf{s} j-1}}^{\rho_{\mathbf{s} i}} I_{n}\left(a, \lambda, \sigma, \rho_{2 j-1} ; y\right) d y \int_{\nu}^{\rho_{\mathbf{s i}}} \frac{\psi_{2 j}(r)}{(r-y)^{1-\sigma}} d r=\int_{\rho_{\mathbf{s} j-1}}^{\rho_{\mathbf{s} j}} \Psi_{2 j}(y) d y$ $\cdot\left\{\frac{\sin \sigma \pi}{\pi} \frac{\Gamma(\sigma)}{\Gamma(1-\sigma)} \sum_{n=0}^{\infty} \widetilde{H}_{n} \triangle_{n}^{2} p_{n}(\lambda-\sigma, \lambda) I_{n}(a, \lambda, \sigma, d ; x) I_{n}\left(a, \lambda, \sigma, \rho_{2 i-1} ; y\right)\right\}$ Q.E.D.

We now discuss reduction of series equations (2.1) and (2.2) to simultaneous Fredholm integral equations of the second kind. Substitution of (3.4) into (2.2) and the use of (3.7) give

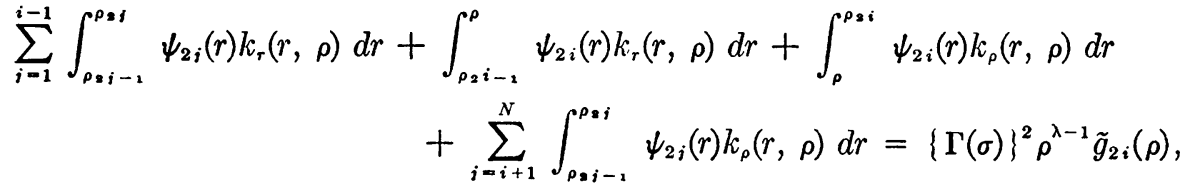

where $\rho_{2 i-1}<\rho<\rho_{2 i}$ and

$\tilde{g}_{2 i}(\rho)=g_{2 i}(\rho)-\sum_{n=0}^{\infty} p_{n}(\lambda-\sigma, \lambda) \tilde{H}_{n} J_{n}(a, \lambda ; \rho) \triangle_{n}^{2} \sum_{j=1}^{N} \int_{\rho_{\mathbf{s} i-1}}^{\rho_{\mathbf{2} i}} \psi_{2 i}(r) r^{\lambda-1} J_{n}(a, \lambda ; r) d r$.

The summation symbol $\sum_{i=h}^{l}$ should be ignored when $h>l$. If we substitute the integral form of $k_{t}(r, \rho)$ into (3.15), then the left-hand side of (3.15) is expressed in terms of a double integral the field of which is shown in Fig. 1. The change of the order of integrations gives

$$
\begin{aligned}
& \sum_{i=1}^{i-1}\left[\int_{0}^{\rho_{3 j-1}} \frac{m(x)}{(\rho-x)^{1-\sigma}} d x \int_{\rho_{3 i-1}}^{\rho_{2 j} j} \frac{\psi_{2 j}(r)}{(r-x)^{1-\sigma}} d r+\int_{\rho_{3 j-1}}^{\rho_{2 \beta}} \frac{m(x)}{(\rho-x)^{1-\sigma}} d x \int_{x}^{\rho_{3 i}} \frac{\psi_{2 j}(r)}{(r-x)^{1-\sigma}} d r\right] \\
& +\int_{0}^{\rho s i-1} \frac{m(x)}{(\rho-x)^{1-\sigma}} d x \int_{\rho_{s i-1}}^{\rho_{3 i}} \frac{\psi_{2 i}(r)}{(r-x)^{1-\sigma}} d r+\int_{\rho_{s i-1}}^{\rho} \frac{m(x)}{(\rho-x)^{1-\sigma}} d x \int_{x}^{\rho_{s i}} \frac{\psi_{2 i}(r)}{(r-x)^{1-\sigma}} d r \\
& +\sum_{i=i+1}^{N}\left[\int_{0}^{\rho, i-1} \frac{m(x)}{(\rho-x)^{1-\sigma}} d x \int_{\rho, i-1}^{\rho_{\mathbf{s i}}} \frac{\psi_{2 i}(r)}{(r-x)^{1-\sigma}} d r\right. \\
& \left.+\int_{\rho, j-1}^{\rho} \frac{m(x)}{(\rho-x)^{1-\sigma}} d x \int_{\rho_{3 i-1}}^{\rho_{s i}} \frac{\psi_{2 j}(r)}{(r-x)^{1-\sigma}} d r\right]=\{\Gamma(\sigma)\}^{2} \rho^{\lambda-1} \tilde{g}_{2 i}(\rho), \quad \rho_{2 i-1}<\rho<\rho_{2 i} .
\end{aligned}
$$

If we substitute (3.5) and (3.9) into (3.17), we obtain

$$
\int_{\rho_{s i-1}}^{\rho} \frac{m(x)}{(\rho-x)^{1-\sigma}}\left\{\Psi_{2 i}(x)+\sum_{i=i+1}^{N} \int_{\rho_{s i-1}}^{\rho s i} T\left(\sigma, \rho_{2 i-1} ; x, y\right) \Psi_{2 j}(y) d y\right\} d x=\chi(\rho),
$$

where

$$
\begin{aligned}
& \chi(\rho)=-\sum_{i=1}^{i-1}\left[\int_{\rho_{i-i-1}}^{\rho_{s i}} \frac{m(y)}{(\rho-y)^{1-\sigma}} \Psi_{2 i}(y) d y\right. \\
& \left.+\int_{0}^{\rho, i-1} \frac{m(\xi)}{(\rho-\xi)^{1-\sigma}} d \xi \int_{\rho_{2 i-1}}^{\rho_{2 i} i} T\left(\sigma, \rho_{2 i-1} ; \xi, y\right) \Psi_{2 i}(y) d y\right]-\int_{0}^{\rho_{2 i-1}} \frac{m(\xi)}{(\rho-\xi)^{1-\sigma}} d \xi \\
& \cdot \sum_{i=i}^{N} \int_{\rho_{j i-1}}^{\rho, f} T\left(\sigma, \rho_{2 i-1} ; \xi, y\right) \Psi_{2 i}(y) d y+\{\Gamma(\sigma)\}^{2} \rho^{\lambda-1} \tilde{g}_{2 i}(\rho) \text {. }
\end{aligned}
$$




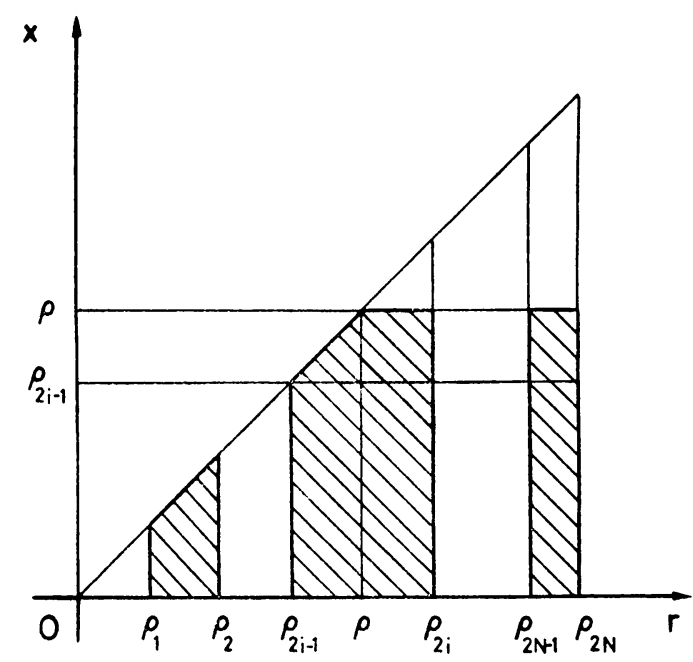

Fig. 1. The field of integration.

By the theory of Volterra integral equations of the first kind, (3.18) is transformed into $m(x) \Psi_{2 i}(x)+m(x) \sum_{i=i+1}^{N} \int_{\rho_{2 i-1}}^{\rho_{3 j}} T\left(\sigma, \rho_{2 i-1} ; x, y\right) \Psi_{2 j}(y) d y=\frac{\sin \sigma \pi}{\pi} \frac{d}{d x} \int_{\rho_{s i-1}}^{x} \frac{\chi(\rho)}{(x-\rho)^{\sigma}} d \rho$.

For the reduction of the right-hand side of (3.20), we define the function $S(\sigma, u, v ; x, y)$ by

$$
\begin{aligned}
S(\sigma, u, v ; x, y) & =\int_{0}^{\min (u, v)} m(\xi) T(\sigma, u ; \xi, x) T(\sigma, v ; \xi, y) d \xi \\
& =\frac{\sin ^{2} \sigma \pi}{\pi^{2}} \frac{1}{(x-u)^{\sigma}(y-v)^{\sigma}} \int_{0}^{\min (u, v)} \frac{m(\xi)(u-\xi)^{\sigma}(v-\xi)^{\sigma}}{(x-\xi)(y-\xi)} d \xi,
\end{aligned}
$$

where $x>u, y>v$. From (3.11) and (3.21), we have for $j<i$

$$
\begin{aligned}
& \frac{\sin \sigma \pi}{\pi} \frac{d}{d x} \int_{\rho_{s i-1}}^{x} \frac{1}{(x-\rho)^{\sigma}} d \rho \int_{\rho_{3 i-1}}^{\rho_{\mathbf{3} i}} \frac{m(y)}{(\rho-y)^{1-\sigma}} \Psi_{2 j}(y) d y \\
& =\int_{\rho_{\mathbf{*} i-1}}^{\rho_{\mathbf{i}} i} m(y) T\left(\sigma, \rho_{2 i-1} ; y, x\right) \Psi_{2 j}(y) d y
\end{aligned}
$$

and

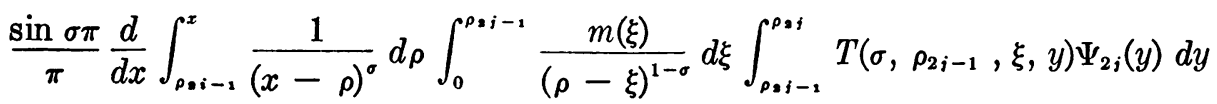

$$
\begin{aligned}
& =\int_{\rho_{i j-1}}^{\rho_{2 i}} \Psi_{2 i}(y) d y \int_{0}^{\rho_{\rho i-1}} m(\xi) T\left(\sigma, \rho_{2 i-1} ; \xi, x\right) T\left(\sigma, \rho_{2 i-1} ; \xi, y\right) d \xi \\
& =\int_{\rho_{\mathbf{s}-1}}^{\rho_{\mathbf{3} i}} S\left(\sigma, \rho_{2 i-1}, \rho_{2 j-1} ; x, y\right) \Psi_{2 j}(y) d y \text {. }
\end{aligned}
$$


Again by the use of (3.11) and (3.21), we have for $i \leqq j$,

$$
\begin{aligned}
& \frac{\sin \sigma \pi}{\pi} \frac{d}{d x} \int_{\rho_{3 i-1}}^{x} \frac{1}{(x-\rho)^{\sigma}} d \rho \int_{0}^{\rho_{s i-1}} \frac{m(\xi)}{(\rho-\xi)^{1-\sigma}} d \xi \int_{\rho_{2 i-1}}^{\rho_{s i}} T\left(\sigma, \rho_{2 j-1} ; \xi, y\right) \Psi_{2 j}(y) d y \\
& =\int_{\rho_{\mathbf{s}_{i-1}}}^{\rho_{2 i}} \Psi_{2 j}(y) d y \int_{0}^{\rho_{2 i-1}} m(\xi) T\left(\sigma, \rho_{2 i-1} ; \xi, x\right) T\left(\sigma, \rho_{2 i-1} ; \xi, y\right) d \xi \\
& =\int_{\rho_{s_{i}-1}}^{\rho_{\boldsymbol{s}_{i}}} S\left(\sigma, \rho_{2 i-1}, \rho_{2 i-1} ; x, y\right) \Psi_{2 i}(y) d y \text {. }
\end{aligned}
$$

From (3.19), (3.20), (3.22), (3.23) and (3.24), we have

$$
\begin{aligned}
& m(x) \Psi_{2 i}(x)+\int_{\rho_{2} i-1}^{\rho_{2 i}} S\left(\sigma, \rho_{2 i-1}, \rho_{2 i-1} ; x, y\right) \Psi_{2 i}(y) d y \\
& +\sum_{i=1}^{i-1} \int_{\rho_{9 ;-1}}^{\rho_{2 i}}\left\{m(y) T\left(\sigma, \rho_{2 i-1} ; y, x\right)+S\left(\sigma, \rho_{2 i-1}, \rho_{2 j-1} ; x, y\right)\right\} \Psi_{2 j}(y) d y \\
& +\sum_{i=i+1}^{N} \int_{\rho_{\mathbf{1} i-2}}^{\rho_{\mathbf{1}}}\left\{m(x) T\left(\sigma, \rho_{2 i-1} ; x, y\right)+S\left(\sigma, \rho_{2 i-1}, \rho_{2 j-1} ; x, y\right)\right\} \Psi_{2 j}(y) d y \\
& =\frac{\Gamma(\sigma)}{\Gamma(1-\sigma)} \frac{d}{d x} \int_{\rho_{21-1}}^{x} \frac{\rho^{\lambda-1} \tilde{g}_{2 i}(\rho)}{(x-\rho)^{\sigma}} d \rho .
\end{aligned}
$$

By using (3.13), terms involving unknown functions $\psi_{2 i}(x)$ on the right-hand side of (3.25) can be transformed into those involving $\Psi_{2 i}(x)$. If we write

$$
\begin{aligned}
& G_{2 i}(x)=\frac{\Gamma(\sigma)}{\Gamma(1-\sigma)} \frac{d}{d x} \int_{\rho, i-1}^{x} \frac{\rho^{\lambda-1} g_{2 i}(\rho)}{(x-\rho)^{\sigma}} d \rho \\
& K_{2 i 2 i}(x, y)=S\left(\sigma, \rho_{2 i-1}, \rho_{2 i-1} ; x, y\right)+\frac{\sin \sigma \pi}{\pi} \frac{\Gamma(\sigma)}{\Gamma(1-\sigma)} \\
& \cdot \sum_{n=0}^{\infty} \tilde{H}_{n} \triangle_{n}^{2} p_{n}(\lambda-\sigma, \lambda) I_{n}\left(a, \lambda, \sigma, \rho_{2 i-1} ; x\right) I_{n}\left(a, \lambda, \sigma, \rho_{2 i-1} ; y\right), \\
& K_{2 i 2 i}(x, y)=m(x) T\left(\sigma, \rho_{2 i-1} ; x, y\right)+S\left(\sigma, \rho_{2 i-1}, \rho_{2 i-1} ; x, y\right)+\frac{\sin \sigma \pi}{\pi} \frac{\Gamma(\sigma)}{\Gamma(1-\sigma)} \\
& \cdot \sum_{n=0}^{\infty} \tilde{H}_{n} \triangle_{n}^{2} p_{n}(\lambda-\sigma, \lambda) I_{n}\left(a, \lambda, \sigma, \rho_{2 i-1} ; x\right) I_{n}\left(a, \lambda, \sigma, \rho_{2 i-1} ; y\right), \quad i<j \\
& K_{2 i 2 j}(x, y)=m(y) T\left(\sigma, \rho_{2 i-1} ; y, x\right)+S\left(\sigma, \rho_{2 i-1}, \rho_{2 j-1} ; x, y\right)+\frac{\sin \sigma \pi}{\pi} \frac{\Gamma(\sigma)}{\Gamma(1-\sigma)} \\
& \cdot \sum_{n=0}^{\infty} \tilde{H}_{n} \triangle_{n}^{2} p_{n}(\lambda-\sigma, \lambda) I_{n}\left(a, \lambda, \sigma, \rho_{2 i-1} ; x\right) I_{n}\left(a, \lambda, \sigma, \rho_{2 j-1} ; y\right), \quad i>j
\end{aligned}
$$

then we finally arrive at the simultaneous Fredholm integral equations of the second kind

$$
\begin{aligned}
m(x) \Psi_{2 i}(x)+\sum_{i=1}^{N} \int_{\rho_{2 i-1}}^{\rho_{2 i}} K_{2 i 2 j}(x, y) \Psi_{2 j}(y) d y=G_{2 i}(x), \rho_{2 i-1} & <x<\rho_{2 i}, \\
i & =1,2, \cdots, N .
\end{aligned}
$$


As to the symmetry of the kernel, the relation $K_{2 i 2 i}(y, x)=K_{2 i 2 j}(x, y)$ should be noted.

4. Triple series equations and quadruple series equations. Triple series equations of the first kind studied by Lowndes [4] can be treated by putting $\rho_{1}=d, \rho_{2}=e, \rho_{3}=1$, $g_{2}(\rho)=f(\rho), \widetilde{H}_{n}=0, N=1$ in (2.1) and (2.2). In this case the integral equation (3.30) is in agreement with that derived by Lowndes.

Triple series equations of the second kind studied by Lowndes [4] can be treated by substituting $\rho_{1}=\rho_{0}=0, \rho_{2}=d, \rho_{3}=e, \rho_{4}=\rho_{5}=1, g_{\varepsilon}(\rho)=g(\rho), g_{4}(\rho)=h(\rho)$, $\widetilde{H}_{n}=0, N=2$ into (2.1) and (2.2). Since $N=2$, we have two integral equations from (3.30). Elimination of $\Psi_{2}(x)$ gives a single integral equation in $\Psi_{\mathbf{i}}(x)$ which is identical with the result of Lowndes. Discussion can be slightly generalized and certain quadruple series equations can be also reduced to a single integral equation. Let

$$
\begin{array}{ll}
\sum_{n=0}^{\infty} D_{n} p_{n}(\lambda-\sigma, \lambda) J_{n}(a, \lambda ; \rho)=g_{2}(\rho), & 0 \leqq \rho<d, \\
\sum_{n=0}^{\infty} D_{n} J_{n}(a, \lambda ; \rho)=0, & d<\rho<e, \\
\sum_{n=0}^{\infty} D_{n} p_{n}(\lambda-\sigma, \lambda) J_{n}(a, \lambda ; \rho)=g_{4}(\rho), & e<\rho<f, \\
\sum_{n=0}^{\infty} D_{n} J_{n}(a, \lambda ; \rho)=0, & f<\rho \leqq 1 .
\end{array}
$$

Series equations can be considered as a special case in which $\rho_{1}=\rho_{0}=0, \rho_{2}=d, \rho_{3}=e$, $\rho_{4}=f, \rho_{5}=1, N=2, \tilde{H}_{n}=0$. From (3.30), we have

$$
\begin{aligned}
& m(x) \Psi_{2}(x)+\int_{0}^{d} K_{22}(x, y) \Psi_{2}(y) d y+\int_{0}^{f} K_{24}(x, y) \Psi_{4}(y) d y=G_{2}(x), \\
& m(x) \Psi_{4}(x)+\int_{0}^{d} K_{42}(x, y) \Psi_{2}(y) d y+\int_{0}^{f} K_{44}(x, y) \Psi_{4}(y) d y=G_{4}(x),
\end{aligned}
$$

where

$$
\begin{aligned}
K_{22}(x, y) & =S(\sigma, 0,0 ; x, y)=0, \\
K_{24}(x, y) & =m(x) T(\sigma, e ; x, y)+S(\sigma, 0, e ; x, y) \\
& =m(x) T(\sigma, e ; x, y), \\
K_{42}(x, y) & =K_{24}(y, x), \\
K_{44}(x, y) & =S(\sigma, e, e ; x, y) .
\end{aligned}
$$

Since $K_{22}(x, y)=0$, we have from (4.2)

$$
\Psi_{2}(\xi)=-\int_{0}^{f} T(\sigma, e ; \xi, y) \Psi_{4}(y) d y+\frac{G_{2}(\xi)}{m(\xi)} .
$$

Substitution of (4.5) into (4.3) and the use of (3.21) give

$$
m(x) \Psi_{4}(x)+\int_{0}^{f} K(x, y) \Psi_{4}(y) d y=-\int_{0}^{d} T(\sigma, e ; \xi, x) G_{2}(\xi) d \xi+G_{4}(x),
$$

where 


$$
\begin{aligned}
K(x, y) & =S(\sigma, e, e ; x, y)-\int_{0}^{d} m(\xi) T(\sigma, e ; \xi, x) T(\sigma, e ; \xi, y) d \xi \\
& =\int_{d}^{e} m(\xi) T(\sigma, e ; \xi, x) T(\sigma, e ; \xi, y) d \xi \\
& =\frac{\sin ^{2} \sigma \pi}{\pi^{2}} \frac{1}{(x-e)^{\sigma}(y-e)^{\sigma}} \int_{d}^{0} \frac{m(\xi)(e-\xi)^{2 \sigma}}{(x-\xi)(y-\xi)} d \xi .
\end{aligned}
$$

Thus the quadruple series equations (4.1) are reduced to a single integral equation. When $f \rightarrow 1$, the series equations (4.1) are reduced to triple series equations of the second kind and the integral equation (4.6) coincides with that derived by Lowndes. When $d \rightarrow 0, g_{2}(\rho)=0$, the series equations (4.1) become triple series equations of the first kind and the integral equation (4.6) is in agreement with the result of Lowndes except for slight changes in notation.

5. $(2 N+1)$ cosine series equations. In order to discuss mixed boundary-value problems of two-dimensional Laplace equations in Sec. 7, trigonometric series equations are studied in detail in Secs. 5 and 6. In this section we consider the following cosine series equations:

$$
\begin{aligned}
& \sum_{n=0}^{\infty} a_{n} \cos \left(n+\frac{1}{2}\right) \theta=0, \quad \theta_{2(i-1)}<\theta<\theta_{2 i-1} \quad(i=1,2, \cdots, N+1), \\
& \sum_{n=0}^{\infty}\left(n+\frac{1}{2}\right)^{-1}\left(1+H_{n}\right) a_{n} \cos \left(n+\frac{1}{2}\right) \theta=f_{2 i}(\theta), \theta_{2 i-1}<\theta<\theta_{2 i} \quad(i=1,2, \cdots, N),
\end{aligned}
$$

where $\theta_{0}=0, \theta_{2 N+1}=\pi$.

For the physical application, it is necessary to compute the quantity

$$
q_{i}=\int_{\theta_{3 i-1}}^{\theta_{2 i}}\left\{\sum_{n=0}^{\infty} a_{n} \cos \left(n+\frac{1}{2}\right) \theta\right\} d \theta .
$$

Let

$$
\begin{gathered}
a=1, \quad \lambda=\frac{3}{2}, \quad \sigma=\frac{1}{2}, \quad \rho=\cos ^{2} \frac{\theta}{2}, \quad \rho_{i}=\cos ^{2} \frac{\theta_{2 N+1-i}}{2}, \\
C_{n}=(-1)^{n}(2 n+1) a_{n}, \quad \tilde{H}_{n}=H_{n} \quad g_{2 i}(\rho)=\frac{f_{2(N+1-i)}\left(2 \cos ^{-1} \sqrt{ } \rho\right)}{\sqrt{ } \rho} .
\end{gathered}
$$

Since

$$
J_{n}\left(1, \frac{3}{2} ; \cos ^{2} \frac{\theta}{2}\right)=(-1)^{n} \frac{1}{2 n+1} \frac{\cos \left(n+\frac{1}{2}\right) \theta}{\cos \frac{\theta}{2}}, \quad p_{n}\left(1, \frac{3}{2}\right)=\frac{1}{n+\frac{1}{2}},
$$

the series equations (5.1) are equivalent to the series equations (2.1) and (2.2). From (5.2), $q_{(N+1-i)}$ is given by

$$
\begin{aligned}
q_{N+1-i} & =\int_{\rho_{3 i-1}}^{\rho_{3 i}}\left\{\sum_{n=0}^{\infty} C_{n} J_{n}(a, \lambda ; \rho)\right\} \frac{d \rho}{(1-\rho)^{1 / 2}} \\
& =\int_{\rho_{x i-1}}^{\rho \geq i} \psi_{2 i}(\rho) d \rho \\
& =\frac{1}{\pi} \int_{\rho_{2 i-1}}^{\rho_{x i}} \frac{\Psi_{2 i}(x)}{\left(x-\rho_{2 i-1}\right)^{1 / 2}} d x .
\end{aligned}
$$


In order to solve the simultaneous integral equations (3.30) numerically, it is necessary to compute kernels. From (3.2), (3.8) and (3.10), we have

$$
\triangle_{n}^{2}=\frac{8}{\pi}\left(n+\frac{1}{2}\right)^{2}, \quad m(x)=1, \quad T(\sigma, u ; x, y)=\frac{1}{\pi} \frac{(u-x)^{1 / 2}}{(y-x)(y-u)^{1 / 2}},
$$

respectively. Elementary computations with (3.21) give

$$
\begin{aligned}
S(\sigma, u, u ; x, y) & =\frac{1}{\pi^{2}((x-u)(y-u))^{1 / 2}} \int_{0}^{u} \frac{u-\xi}{(x-\xi)(y-\xi)} d \xi \\
& =\frac{1}{\pi^{2}} \cdot \frac{1}{x-y}\left\{\left(\frac{x-u}{y-u}\right)^{1 / 2} \log \left|\frac{x}{x-u}\right|-\left(\frac{y-u}{x-u}\right)^{1 / 2} \log \left|\frac{y}{y-u}\right|\right\} .
\end{aligned}
$$

where $x, y>u$. Functions $S(\sigma, u, v ; x, y)$ for $u<x<v<y$ can be expressed by elementary functions but its derivation is not so obvious so that we describe it briefly. From (3.21) we have

$$
\begin{aligned}
S(\sigma, u, v ; x, y) & =\frac{1}{\pi^{2}((x-u)(y-v))^{1 / 2}} \frac{1}{y-x} \int_{0}^{u}\left(\frac{1}{x-\xi}-\frac{1}{y-\xi}\right)(u-\xi)^{1 / 2}(v-\xi)^{1 / 2} d \xi \\
& =\frac{1}{\pi^{2}((x-u)(y-v))^{1 / 2}} \frac{1}{y-x}\{M(x)-M(y)\}
\end{aligned}
$$

where

$$
M(z)=\int_{0}^{u} \frac{(u-\xi)^{1 / 2}(v-\xi)^{1 / 2}}{z-\xi} d \xi .
$$

Using the variable transformation $w=(u-\xi)^{1 / 2} /(v-\xi)^{1 / 2},(5.8)$ is reduced to

$$
M(z)=-\frac{2(v-u)^{2}}{z-v} \int_{0}^{(u / v)^{1 / s}} \frac{w^{2}}{\left(w^{2}-c\right)\left(w^{2}-1\right)^{2}} d w,
$$

where

$$
c=(z-u) /(z-v) .
$$

After some elementary computations, we have

$$
M(z)=z \log \left|\frac{\sqrt{ } v-\sqrt{ } u}{\sqrt{ } v+\sqrt{ } u}\right|-2(z-u) \int_{0}^{(u / v))^{1 / z}} \frac{d w}{w^{2}-c}+M_{0},
$$

where $M_{0}$ is independent of $z$ and given by

$$
M_{0}=-(u+v) \int_{0}^{(u / v)^{2 / 2}} \frac{d \omega}{w^{2}-1}+\frac{1}{2}(v-u) \int_{0}^{(u / v)^{1 / 2}}\left\{\frac{1}{(w-1)^{2}}+\frac{1}{(w+1)^{2}}\right\} d w .
$$

The definite integral on the right-hand side of (5.11) can be evaluated by the formula

$$
\int_{0}^{w_{0}} \frac{d w}{w^{2}-c}= \begin{cases}\frac{1}{2 \sqrt{ } c} \log \left|\frac{w_{0}-\sqrt{ } c}{w_{0}+\sqrt{ } c}\right| & (c>0) \\ \frac{1}{\sqrt{ }|c|} \tan ^{-1} \frac{w_{0}}{\sqrt{ }|c|} & (c<0) .\end{cases}
$$


Since $u<x<v<y$ and therefore, for $z=x, c=-(x-u) /(v-x)<0$ and for $z=y, c=(y-u) /(y-v)>0$, so that we find from (5.7), (5.10), (5.11) and (5.13)

$$
\begin{aligned}
S(\sigma, u, v ; x, y)= & -\frac{1}{\pi^{2}((x-u)(y-v))^{1 / 2}} \log \left|\frac{\sqrt{ } v-\sqrt{ } u}{\sqrt{ } v+\sqrt{ } u}\right| \\
& +\frac{2}{\pi^{2}} \cdot \frac{1}{x-y}\left(\frac{v-x}{y-v}\right)^{1 / 2} \tan ^{-1}\left(\left(\frac{u}{x-u}\right)^{1 / 2}\left(\frac{v-x}{v}\right)^{1 / 2}\right) \\
& -\frac{1}{\pi^{2}} \frac{1}{x-y}\left(\frac{y-u}{x-u}\right)^{1 / 2} \log \left|\frac{\left(\frac{y-u}{u}\right)^{1 / 2}}{\left(\frac{y-u}{u}\right)^{1 / 2}}+\frac{\left(\frac{y-v}{v}\right)^{1 / 2}}{\left(\frac{y-v}{v}\right)^{1 / 2}}\right| .
\end{aligned}
$$

To complete the computation of kernels, it suffices to show how to compute the function $I_{n}(a, \lambda, \sigma, d ; x)$. If we write

$$
\tilde{I}(\beta ; u, n)=(-1)^{n} \frac{2}{\pi}\left(n+\frac{1}{2}\right)^{1 / 2} I_{n}\left(1, \frac{3}{2}, \frac{1}{2}, \cos ^{2} \frac{\beta}{2} ; \cos ^{2} \frac{u}{2}\right),
$$

then we find

$$
\tilde{I}(\beta ; u, n)=\frac{\sqrt{ } 2}{\pi}\left(n+\frac{1}{2}\right)^{-1 / 2} \frac{\cos \left(n+\frac{1}{2}\right) \beta}{(\cos u-\cos \beta)^{1 / 2}}+\left(n+\frac{1}{2}\right)^{1 / 2} R_{n}(\beta ; u),
$$

where

$$
R_{n}(\beta ; u)=\frac{\sqrt{ } 2}{\pi} \int_{u}^{\beta} \frac{\sin \left(n+\frac{1}{2}\right) \varphi}{(\cos u-\cos \varphi)^{1 / 2}} d \varphi .
$$

The $I(\beta ; u, n)$ and $R_{n}(\beta ; u)$ are identical with $I(u, n)$ and $R_{n}(u)$ with $\beta=c$ defined by the authors [3]. $R_{n}(\beta ; u)$ can be computed by the recurrence relation similar to that for the Legendre polynomials:

$R_{0}(\beta ; u)=1-\frac{2}{\pi} \sin ^{-1}\left(\frac{\cos \frac{\beta}{2}}{\cos \frac{u}{2}}\right)$,

$R_{1}(\beta ; u)=R_{0}(\beta ; u) \cos u+\frac{2 \sqrt{ } 2}{\pi} \cos \frac{\beta}{2}(\cos u-\cos \beta)^{1 / 2}$,

$(n+1) R_{n+1}(\beta ; u)-(2 n+1) R_{n}(\beta ; u) \cos u+n R_{n-1}(\beta ; u)$

$$
=\frac{2 \sqrt{ } 2}{\pi} \cos \left(n+\frac{1}{2}\right) \beta(\cos u-\cos \beta)^{1 / 2}, \quad n \geqq 1 .
$$

We finally find for kernels

$$
\begin{aligned}
K_{2 i 2 i}(x, y)= & \frac{1}{\pi^{2}} \frac{1}{x-y}\left\{\left(\frac{x-\rho_{2 i-1}}{y-\rho_{2 i-1}}\right)^{1 / 2} \log \left|\frac{x}{x-\rho_{2 i-1}}\right|-\left(\frac{y-\rho_{2 i-1}}{x-\rho_{2 i-1}}\right)^{1 / 2} \log \left|\frac{y}{y-\rho_{2 i-1}}\right|\right\} \\
+ & 2 \sum_{n=0}^{\infty} H_{n} \tilde{I}\left(2 \cos ^{-1}\left(\rho_{2 i-1}\right)^{1 / 2} ; 2 \cos ^{-1} \sqrt{ } x, n\right) \\
& \cdot I\left(2 \cos ^{-1}\left(\rho_{2 i-1}\right)^{1 / 2} ; 2 \cos ^{-1} \sqrt{ } y, n\right),
\end{aligned}
$$




$$
\begin{aligned}
& K_{2 i 2 i}(x, y)=\frac{1}{\pi} \frac{\left(\rho_{2 j-1}-x\right)^{1 / 2}}{(y-x)\left(y-\rho_{2 j-1}\right)^{1 / 2}} \\
& -\frac{1}{\pi^{2}\left(\left(x-\rho_{2 i-1}\right)\left(y-\rho_{2 i-1}\right)\right)^{1 / 2}} \log \left|\frac{\left(\rho_{2 i-1}\right)^{1 / 2}-\left(\rho_{2 i-1}\right)^{1 / 2}}{\left(\rho_{2 i-1}\right)^{1 / 2}+\left(\rho_{2 i-1}\right)^{1 / 2}}\right| \\
& +\frac{2}{\pi^{2}} \frac{1}{x-y}\left(\frac{\rho_{2 j-1}-x}{y-\rho_{2 j-1}}\right)^{1 / 2} \tan ^{-1}\left(\left(\frac{\rho_{2 i-1}}{x-\rho_{2 i-1}}\right)^{1 / 2}\left(\frac{\rho_{2 j-1}-x}{\rho_{2 j-1}}\right)^{1 / 2}\right) \\
& -\frac{1}{\pi^{2}} \frac{1}{x-y}\left(\frac{y-\rho_{2 i-1}}{x-\rho_{2 i-1}}\right)^{1 / 2} \log \left|\frac{\left(\frac{y-\rho_{2 i-1}}{\rho_{2 i-1}}\right)^{1 / 2}-\left(\frac{y-\rho_{2 i-1}}{\rho_{2 i-1}}\right)^{1 / 2}}{\left(\frac{y-\rho_{2 i-1}}{\rho_{2 i-1}}\right)^{1 / 2}+\left(\frac{y-\rho_{2 i-1}}{\rho_{2 i-1}}\right)^{1 / 2}}\right| \\
& +2 \sum_{n=0}^{\infty} H_{n} \tilde{I}\left(2 \cos ^{-1}\left(\rho_{2 i-1}\right)^{1 / 2} ; 2 \cos ^{-1} \sqrt{ } x, n\right) \\
& . \widetilde{I}\left(2 \cos ^{-1}\left(\rho_{2 j-1}\right)^{1 / 2} ; 2 \cos ^{-1} \sqrt{ } y, n\right), \quad j>i, \\
& K_{2 i 2 j}(x, y)=K_{2 j 2 i}(y, x) \quad j<i .
\end{aligned}
$$

The value of $K_{2 i 2 i}(x, x)$ can be computed by the L'Hospital theorem.

For numerical computation it was found convenient to write $x=\rho_{2 i-1} \sec ^{2} \xi$ when $\rho_{2 i-1} \neq 0$ or $x=\cos ^{2}(\xi / 2)$ when $\rho_{2 i-1}=0$. This can be illustrated by the example of triple cosine series equations. For simplicity we consider the case $f_{2}(\theta)=1$. This problem will play an important role in the analysis of boundary-value problems of the Laplace equation in Sec. 7. If we write

$$
\begin{gathered}
x=\rho_{1} \sec ^{2} \xi, \quad 0 \leqq \xi \leqq \sec ^{-1}\left(\frac{\cos \left(\theta_{1} / 2\right)}{\cos \left(\theta_{2} / 2\right)}\right) \equiv \xi_{0}, \\
\Psi_{2}(x)=\frac{1}{\sqrt{ } \rho_{1}} E(\xi) \cos ^{2} \xi,
\end{gathered}
$$

then from (3.30) and (5.20) we finally obtain the following integral equation:

$$
\begin{gathered}
\sin \xi \cos \xi E(\xi)+\int_{0}^{\xi \bullet} L_{\text {even }}(\xi, \eta) E(\eta) d \eta=1, \\
L_{\text {even }}(\xi, \eta)=\frac{4}{\pi^{2}} \frac{\tan ^{2} \xi \log (\sin \xi)-\tan ^{2} \eta \log (\sin \eta)}{\sec ^{2} \eta-\sec ^{2} \xi} \\
+4 \rho_{1} \tan \xi \tan \eta \sum_{n=0}^{\infty} H_{n} I\left(2 \cos ^{-1} \sqrt{ } \rho_{1} ; 2 \cos ^{-1}\left(\sqrt{ } \rho_{1} \sec \xi\right), n\right) \\
\cdot I\left(2 \cos ^{-1} \sqrt{ } \rho_{1} ; 2 \cos ^{-1}\left(\sqrt{ } \rho_{1} \sec \eta\right), n\right) .
\end{gathered}
$$

The value of $L_{\text {even }}(\xi, \eta)$ for $\xi=\eta$ can be computed using L'Hospital's theorem. The value of $q_{1}$ can be computed by

$$
q_{1}=\frac{2}{\pi} \int_{0}^{\xi_{0}} E(\xi) d \xi .
$$

6. $(2 N+1)$ sine series equations. In this section we discuss the sine series equations 


$$
\begin{array}{ll}
\sum_{n=1}^{\infty} a_{n} \sin n \theta=0, \theta_{2(i-1)}<\theta<\theta_{2 i-1} & (i=1,2, \cdots, N+1), \\
\sum_{n=1}^{\infty} n^{-1}\left(1+H_{n}\right) a_{n} \sin n \theta=f_{2 i}(\theta), \theta_{2 i-1}<\theta<\theta_{2 i} & (i=1,2, \cdots, N),
\end{array}
$$

where $\theta_{0}=0, \theta_{2 N+1}=\pi$. For the physical application, it is necessary to compute the quantity

$$
q_{i}=\int_{\theta_{2 i-1}}^{\theta_{2 i}}\left\{\sum_{n=1}^{\infty} a_{n} \sin n \theta\right\} d \theta .
$$

Let $a=2, \lambda=3 / 2, \sigma=1 / 2, \rho=\sin ^{2}(\theta / 2), \rho_{i}=\sin ^{2}\left(\theta_{i} / 2\right), C_{n}=(n+1) a_{n+1}, \tilde{H}_{n}=$ $H_{n+1}, g_{2 i}(\rho)=\left(f_{2 i}\left(2 \sin ^{-1} \sqrt{ } \rho\right) / 2(\rho(1-\rho))^{1 / 2}\right.$. Since

$$
J_{n}\left(2, \frac{3}{2} ; \sin ^{2} \frac{\theta}{2}\right)=\frac{1}{n+1} \frac{\sin (n+1) \theta}{\sin \theta}, \quad p_{n}\left(1, \frac{3}{2}\right)=\frac{1}{n+1},
$$

the series equations (6.1) are treated as a special case of the series equations (2.1) and (2.2). From (6.2), we find

$$
\begin{aligned}
q_{i} & =2 \int_{\rho_{3 i-1}}^{\rho_{3 i}}\left\{\sum_{n=0}^{\infty} C_{n} J_{n}(a, \lambda ; \rho)\right\} d \rho \\
& =2 \int_{\rho_{2 i-1}}^{\rho_{3 i}} \frac{\psi_{2 i}(\rho)}{(1-\rho)^{1 / 2}} d \rho \\
& =2 \int_{\rho_{2 i-1}}^{\rho_{3 i}} \frac{1}{(1-\rho)^{1 / 2}}\left\{-\frac{1}{\pi} \frac{d}{d \rho} \int_{\rho}^{\rho \leq i} \frac{\Psi_{2 i}(x)}{(x-\rho)^{1 / 2}} d x\right\} d \rho \\
& =\frac{2}{\pi} \int_{\rho_{2 i-1}}^{\rho_{1 i}} \frac{\left(1-\rho_{2 i-1}\right)^{1 / 2}}{(1-x)\left(x-\rho_{2 i-1}\right)^{1 / 2}} \Psi_{2 i}(x) d x .
\end{aligned}
$$

We now give kernels of integral equations for sine series equations. From (3.2), (3.8) and (3.10) we have

$$
\Delta_{n}^{2}=\frac{8}{\pi}(n+1)^{2}, \quad m(x)=\frac{1}{1-x}, \quad T(\sigma, u ; x, y)=\frac{1}{\pi} \frac{(u-x)^{1 / 2}}{(y-x)(y-u)^{1 / 2}},
$$

respectively. We find from (3.21)

$$
\begin{aligned}
& S(\sigma, u ; u ; x, y) \\
& =\frac{1}{\pi^{2}((x-u)(y-u))^{1 / 2}} \int_{0}^{u} \frac{u-\xi}{(1-\xi)(x-\xi)(y-\xi)} d \xi \\
& =\frac{1}{\pi^{2}((x-u)(y-u))^{1 / 2}} \frac{(1-u)}{(1-x)(1-y)} \log |1-u| \\
& \quad+\frac{1}{\pi^{2}} \frac{1}{x-y}\left\{\frac{1}{1-x}\left(\frac{x-u}{y-u}\right)^{1 / 2} \log \left|\frac{x}{x-u}\right|-\frac{1}{1-y}\left(\frac{y-u}{x-u}\right)^{1 / 2} \log \left|\frac{y}{y-u}\right|\right\},
\end{aligned}
$$

where $x, y>u$. The function $S(\sigma, u, v ; x, y)$ for $u<x<v<y$ can be evaluated in a way similar to that for the cosine series equations. Some elementary algebra with (3.21) gives 
$S(\sigma, u, v ; x, y)$

$$
\begin{aligned}
& =\frac{1}{\pi^{2}((x-u)(y-v))^{1 / 2}} \int_{0}^{u} \frac{(u-\xi)^{1 / 2}(v-\xi)^{1 / 2}}{(1-\xi)(x-\xi)(y-\xi)} d \xi \\
& =\frac{1}{\pi^{2}((x-u)(y-v))^{1 / 2}}\left\{\frac{M(1)}{(1-x)(1-y)}+\frac{M(x)}{(y-x)(1-x)}+\frac{M(y)}{(x-y)(1-y)}\right\},
\end{aligned}
$$

where $M(z)$ is defined by (5.11). From (5.10), (5.13) and (6.7), we find $S(\sigma, u, v ; x, y)$

$$
\begin{aligned}
= & -\frac{(1-u)^{1 / 2}(1-v)^{1 / 2}}{\pi^{2}((x-u)(y-v)(1-x)(1-y))^{1 / 2}} \log \left|\frac{(u(1-v))^{1 / 2}-(v(1-u))^{1 / 2}}{(u(1-v))^{1 / 2}+(v(1-u))^{1 / 2}}\right| \\
& +\frac{2}{\pi^{2}} \frac{1}{(x-y)(1-x)}\left(\frac{v-x}{y-v}\right)^{1 / 2} \tan ^{-1}\left(\left(\frac{u}{x-u}\right)^{1 / 2}\left(\frac{v-x}{v}\right)^{1 / 2}\right) \\
& -\frac{1}{\pi^{2}} \frac{1}{(x-y)(1-y)}\left(\frac{y-u}{x-u}\right)^{1 / 2} \log \left|\frac{\left(\frac{y-u}{u}\right)^{1 / 2}-\left(\frac{y-v}{v}\right)^{1 / 2}}{\left(\frac{y-u}{u}\right)^{1 / 2}+\left(\frac{y-v}{v}\right)^{1 / 2}}\right| .
\end{aligned}
$$

If we define

$$
\widetilde{Q}_{n}(\alpha ; u)=\frac{2}{\pi} I_{n-1}\left(2, \frac{3}{2}, \frac{1}{2}, \sin ^{2} \frac{\alpha}{2} ; \sin ^{2} \frac{u}{2}\right),
$$

we have from (3.27), (3.28) and (3.29)

$$
\begin{aligned}
& K_{2 i 2 i}(x, y)=\frac{1}{\pi^{2}} \frac{1}{\left(\left(x-\rho_{2 i-1}\right)\left(y-\rho_{2 i-1}\right)\right)^{1 / 2}} \frac{\left(1-\rho_{2 i-1}\right)}{(1-x)(1-y)} \log \left|1-\rho_{2 i-1}\right| \\
& +\frac{1}{\pi^{2}} \frac{1}{x-y}\left\{\frac{1}{1-x}\left(\frac{x-\rho_{2 i-1}}{y-\rho_{2 i-1}}\right)^{1 / 2} \log \left|\frac{x}{x-\rho_{2 i-1}}\right|\right. \\
& \left.-\frac{1}{1-y}\left(\frac{y-\rho_{2 i-1}}{x-\rho_{2 i-1}}\right)^{1 / 2} \log \left|\frac{y}{y-\rho_{2 i-1}}\right|\right\} \\
& \left.+\sum_{n=1}^{\infty} 2 n H_{n} \widetilde{Q}_{n}\left(2 \sin ^{-1}\left(\rho_{2 i-1}\right)^{1 / 2} ; 2 \sin ^{-1} \sqrt{ } x\right) \widetilde{Q}_{n}\left(2 \sin ^{-1}\left(\rho_{2 i-1}\right)^{1 / 2} ; 2 \sin ^{-1} \sqrt{ } y\right),\right\} \\
& K_{2 i 2 j}(x, y)=\frac{\left(\rho_{2 j-1}-x\right)^{1 / 2}}{\pi(1-x)(y-x)\left(y-\rho_{2 i-1}\right)^{1 / 2}}-S\left(\sigma, \rho_{2 i-1}, \rho_{2 j-1} ; x, y\right) \\
& +\sum_{n=1}^{\infty} 2 n H_{n} \widetilde{Q}_{n}\left(2 \sin ^{-1}\left(\rho_{2 i-1}\right)^{1 / 2} ; 2 \sin ^{-1} \sqrt{ } x\right) \widetilde{Q}_{n}\left(2 \sin ^{-1}\left(\rho_{2 i-1}\right)^{1 / 2} ; 2 \sin ^{-1} \sqrt{ } y\right), \\
& \text { for } i<j \text {, } \\
& K_{2 i 2 j}(x, y)=K_{2 j 2 i}(y, x) \\
& \text { for } i>j \text {, }
\end{aligned}
$$

where $S\left(\sigma, \rho_{2 i-1}, \rho_{2 j-1} ; x, y\right)$ is given by (6.8). The value of $K_{2 i 2 i}(x, x)$ can be computed by L'Hospital's theorem.

In order to compute $Q_{n}(\alpha ; u)$ we derive a recurrence formula. From (6.9) we find 


$$
\widetilde{Q}_{n}(\alpha ; u)=\frac{1}{n \sin u} \frac{d}{d u} \frac{\sqrt{ } 2}{\pi} \int_{\alpha}^{u} \frac{\cos \left(n-\frac{1}{2}\right) \varphi-\cos \left(n+\frac{1}{2}\right) \varphi}{(\cos \varphi-\cos u)^{1 / 2}} d \varphi .
$$

We define the function $\tilde{R}_{n}(\alpha ; u)$ by

$$
\tilde{R}_{n}(\alpha ; u)=\frac{\sqrt{ } 2}{\pi} \int_{\alpha}^{u} \frac{\cos \left(n+\frac{1}{2}\right) \varphi}{(\cos \varphi-\cos u)^{1 / 2}} d \varphi ;
$$

then we have the following relation between $\widetilde{R}_{n}(\alpha ; u)$ and $R_{n}(\beta ; u)$ :

$$
\tilde{R}_{n}(\alpha ; u)=(-1)^{n} R_{n}(\pi-\alpha ; \pi-u) .
$$

From (6.11), we have

$$
\begin{aligned}
(n+1) \tilde{Q}_{n+1}(\alpha ; u)+n \widetilde{Q}_{n}(\alpha ; u) & =\frac{1}{\sin u} \frac{d}{d u} \frac{\sqrt{ } 2}{\pi} \int_{\alpha}^{u} \frac{\cos \left(n-\frac{1}{2}\right) \varphi-\cos \left(n+\frac{3}{2}\right) \varphi}{(\cos \varphi-\cos u)^{1 / 2}} d \varphi \\
& =\frac{1}{\sin u} \frac{d}{d u} \frac{\sqrt{ } 2}{\pi} \int_{\alpha}^{u} \frac{2 \sin \varphi \sin \left(n+\frac{1}{2}\right) \varphi}{(\cos \varphi-\cos u)^{1 / 2}} d \varphi .
\end{aligned}
$$

Applying the integration by parts to (6.14), we have

$$
\begin{aligned}
(n+1) \widetilde{Q}_{n+1}(\alpha ; u) & +n \widetilde{Q}_{n}(\alpha ; u) \\
& =(2 n+1) \tilde{R}_{n}(\alpha ; u)+\frac{2 \sqrt{ } 2}{\pi} \frac{\sin \left(n+\frac{1}{2}\right) \alpha}{(\cos \alpha-\cos u)^{1 / 2}}, \quad n \geqq 1 .
\end{aligned}
$$

By some elementary algebra, we have

$$
\tilde{Q}_{1}(\alpha ; u)=\tilde{R}_{0}(\alpha ; u)+\frac{2 \sqrt{ } 2}{\pi}\left(\sin \frac{1}{2} \alpha /(\cos \alpha-\cos u)^{1 / 2}\right) .
$$

If we can compute $\tilde{R}_{n}(\alpha ; u)$, then $\widetilde{Q}_{n}(\alpha ; u)$ can be computed by the recurrence formulas (6.15) and (6.16). Now the relation (6.13) should be recalled. As far as the function $R_{n}(\beta ; u)$ is concerned, we gave the recurrence formula (5.18).

For the numerical computation it was found convenient to write $x=\rho_{2 i-1} \sec ^{2} \xi$ provided that $\rho_{2 i-1} \neq 0$. This is illustrated by the example of triple series equations. For simplicity we consider $f_{2}(\theta)=1$. This problem is important for the analysis of mixed boundary-value problems of the Laplace equation which is discussed in Sec. 7. If we write

$$
\begin{gathered}
x=\rho_{1} \sec ^{2} \xi, \quad 0 \leqq \xi \leqq \sec ^{-1}\left(\sin \frac{\theta_{2}}{2} / \sin \frac{\theta_{1}}{2}\right) \equiv \xi_{0}, \\
\frac{\Psi_{2}(x)}{1-x}=\frac{1}{2} \cot \frac{\theta_{1}}{2} E(\xi) \cos ^{2} \xi,
\end{gathered}
$$

then from (3.30) and (6.17), we finally obtain the following integral equation:

$$
\begin{gathered}
\sin \xi \cos \xi\left(1-\rho_{1} \sec ^{2} \xi\right) E(\xi)+\int_{0}^{\xi_{0}} L_{\text {odd }}(\xi, \eta) E(\eta) d \eta=1, \\
L_{\text {odd }}(\xi, \eta)=\frac{2}{\pi^{2}}\left(1-\rho_{1}\right) \log \left(1-\rho_{1}\right)+\frac{4 \rho_{1}}{\pi^{2}} \frac{\sin ^{2} \xi \log (\sin \xi)-\sin ^{2} \eta \log (\sin \eta)}{\sin ^{2} \xi-\sin ^{2} \eta} \\
+\frac{4}{\pi^{2}} \frac{\tan ^{2} \xi \log (\sin \xi)-\tan ^{2} \eta \log (\sin \eta)}{\sec ^{2} \eta-\sec ^{2} \xi}
\end{gathered}
$$




$$
\begin{aligned}
& +4 \rho_{1}\left(1-\rho_{1} \sec ^{2} \xi\right)\left(1-\rho_{1} \sec ^{2} \eta\right) \tan \xi \tan \eta \\
& \cdot \sum_{n=1}^{\infty} n H_{n} \widetilde{Q}_{n}\left(\theta_{1} ; 2 \sin ^{-1}\left(\sqrt{ } \rho_{1} \sec \xi\right)\right) \widetilde{Q}_{n}\left(\theta_{1} ; 2 \sin ^{-1}\left(\sqrt{ } \rho_{1} \sec \eta\right)\right) .
\end{aligned}
$$

The value of $L_{\text {odd }}(\xi, \eta)$ for $\xi=\eta$ can be computed using L'Hospital's theorem. From (6.4) and (6.17), the quantity $q_{1}$ is given by

$$
q_{1}=\frac{2}{\pi}\left(1-\rho_{1}\right) \int_{0}^{\xi_{\bullet}} E(\xi) d \xi .
$$

7. Application to a mixed boundary-value problem of the Laplace equation. Using the method in the preceding section, we discuss a mixed boundary-value problem of the Laplace equation. The problem arises in electrostatics and has an application to computation of the Maxwellian capacitance matrix of a transmission line system [1], [2], [3]. Let us consider the transmission line system whose cross-section is shown in Fig. 2. We assume the symmetry of the line structure in respect to $x=0$. We further assume that the potential of the outer waveguide $\Gamma$ is maintained zero. The Maxwellian capacitance matrix of the transmission line system is defined in the following way. Let $q_{1}, q_{2}, \cdots, q_{2 N}$ be the charge per unit length on the strips of the transmission line system with $v_{1}, v_{2}, \cdots, v_{2 N}$ the corresponding potentials. The charge and the potential are related by the Maxwellian capacitance matrix $C=\left(C_{i j}\right)$ as

$$
\mathrm{q}=\mathrm{C} \mathbf{v} \text {, }
$$

where $\mathbf{q}=\left(q_{1}, q_{2}, \cdots, q_{2 N}\right)^{t}$ and $\mathbf{v}=\left(v_{1}, v_{2}, \cdots, v_{2 N}\right)^{t}$. It is well known that $C$ is a symmetric matrix [2].

The matrix element $C_{i i}$ can be computed by solving boundary-value problems of the Laplace equation. We denote the potential in the waveguide by

$$
\begin{aligned}
V & =V_{1}, \quad 0 \leqq y \leqq h_{1}, \\
& =V_{2}, \quad h_{1} \leqq y \leqq h_{1}+h_{2}, \\
& =V_{\mathbf{z}}, h_{1}+h_{2} \leqq y \leqq h_{1}+h_{2}+h_{2} .
\end{aligned}
$$

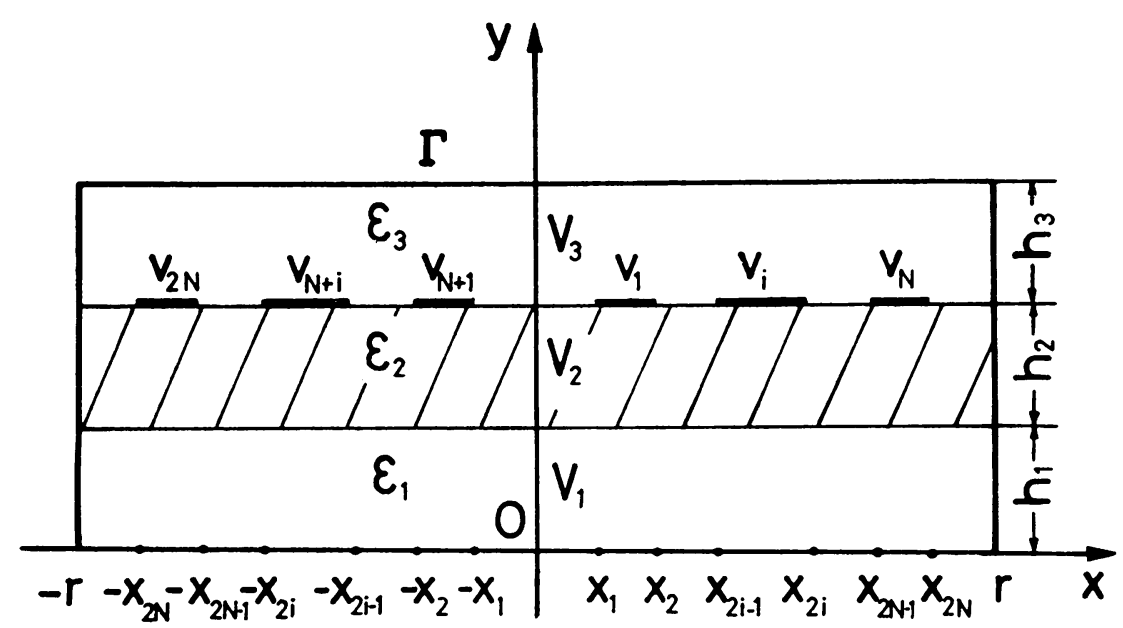

FIG. 2. Symmetric multiconductor transmission line system. 
$V$ satisfies the Laplace equation in the appropriate region and the continuity condition

$$
\begin{aligned}
V_{1} & =V_{2}, & y & =h_{1}, \\
\epsilon_{1} \frac{\partial V_{1}}{\partial y} & =\epsilon_{2} \frac{\partial V_{2}}{\partial y}, & y & =h_{1}, \\
V_{2} & =V_{3}, & y & =h_{1}+h_{2},
\end{aligned}
$$

where $\epsilon_{i}$ denotes the dielectric constant of the medium filled in the relevant region. Boundary conditions to be satisfied by $V_{i}$ are as follows:

$$
\begin{aligned}
V_{i} & =0 \quad(i=1,2,3) \quad \text { on } \quad \Gamma, \\
\epsilon_{2} \frac{\partial V_{2}}{\partial y} & =\epsilon_{3} \frac{\partial V_{3}}{\partial y},\left\{\begin{array}{l}
y=h_{1}+h_{2}, \quad x_{2 i-2}<x<x_{2 i-1} \\
y=h_{1}+h_{2},-x_{2 i-1}<x<-x_{2 i-2}
\end{array} \quad(i=1,2, \cdots, N+1),\right. \\
V_{2} & =v_{i}, \quad y=h_{1}+h_{2}, x_{2 i-1}<x<x_{2 i} \\
V_{2} & =v_{i+N}, \quad y=h_{1}+h_{2},-x_{2 i}<x<-x_{2 i-1}
\end{aligned} \quad(i=1,2, \cdots, N),
$$

where $x_{0}=0, x_{2 N+1}=r$. The charge on the strip conductor is

$$
q_{i}=\int_{x_{i ;-1}}^{x_{y i}} \sigma(x) d x, \quad q_{i+N}=\int_{-x_{i i}}^{-x_{* i-1}} \sigma(x) d x \quad(i=1,2, \cdots, N)
$$

where

$$
\sigma(x)=\epsilon_{2} \frac{\partial V_{2}}{\partial y}-\epsilon_{3} \frac{\partial V_{3}}{\partial y}, \quad y=h_{1}+h_{2} .
$$

If $\boldsymbol{\nabla}$ is given, we can compute $q$ by solving the above boundary-value problem. In order to compute the Maxwellian capacitance matrix $C$, we consider the following two kinds of boundary-value problems.

$$
\begin{aligned}
& v_{k}=v_{k+N}=0, \quad k=1,2, \cdots, j-1, j+1, \cdots, N, \\
& v_{i}=v_{i+N}=1 .
\end{aligned}
$$

Solving this boundary-value problem, we obtain

$$
C_{i j}+C_{i+N}=q_{0 .},
$$

where $q_{o i} \equiv q_{i}=q_{i+N}$.

$$
\begin{aligned}
& v_{k}=v_{k+N}=0, \quad k=1,2, \cdots, j-1, j+1, \cdots, N, \\
& v_{j}=-v_{i+N}=1 .
\end{aligned}
$$

Solving this boundary-value problem, we obtain

$$
C_{i j}-C_{i j+N}=q_{0 i} \text {. }
$$

where $q_{o i} \equiv q_{i}=-q_{t+N}$. From (7.10) and (7.12), we have

$$
\begin{aligned}
C_{i j}=C_{i i} & =\frac{1}{2}\left(q_{o i}+q_{o i}\right), \quad(i, j=1,2, \cdots, N), \\
C_{i i+N}=C_{i+N i} & =\frac{1}{2}\left(q_{0 i}-q_{o i}\right),
\end{aligned}
$$


where use is made of the symmetry of the matrix $C$. Problems (a) and (b) can be reduced to trigonometric series equations by a method similar to that used for a single strip line problem [3]. We define $\lambda_{n}, L_{n}$ and $H_{n}$ by

$$
\begin{gathered}
\lambda_{n}=\left(n+\frac{1}{2}\right) \pi / r, \quad n=0,1,2, \cdots \quad \text { for problem (a), } \\
\lambda_{n}=n \pi / r \quad n=1,2, \cdots \quad \text { for problem (b), } \\
L_{n}=e^{-2 \lambda_{n} h_{2}}\left(1+\kappa_{12} e^{2 \lambda n h_{2}}\right) /\left(1+\kappa_{12} e^{-2 \lambda_{n} h_{2}}\right), \\
H_{n}=-\frac{L_{n} e^{-2 \lambda_{n} h_{s}}+e^{-2 \lambda_{n} h_{s}}-2 L_{n} e^{-2 \lambda_{n}\left(h_{s}+h_{3}\right)}+\kappa_{23}\left(L_{n} e^{-2 \lambda_{n} h_{s}}-e^{-2 \lambda_{n} h_{2}}\right)}{1-L_{n} e^{-2 \lambda_{n}\left(h_{s}+h_{s}\right)}+\kappa_{23}\left(L_{n} e^{-2 \lambda_{n} h_{s}}-e^{-2 \lambda_{n} h_{s}}\right)},
\end{gathered}
$$

respectively, where $k_{i i}=\left(\epsilon_{i}-\epsilon_{i}\right) /\left(\epsilon_{i}+\epsilon_{i}\right)$. Problem (a) is reduced to

$\sum_{n=0}^{\infty} a_{n} \cos \left(n+\frac{1}{2}\right) \theta=0, \quad \theta_{2 i-2}<\theta<\theta_{2 i-1}, \quad i=1, \cdots, N+1$,

$\sum_{n=0}^{\infty}\left(n+\frac{1}{2}\right)^{-1}\left(1+H_{n}\right) a_{n} \cos \left(n+\frac{1}{2}\right) \theta=v_{i}, \quad \theta_{2 i-1}<\theta<\theta_{2 i}, \quad i=1, \cdots, N,(7.1$

where $\theta_{t}=\pi x_{i} / r$ and the charge $q_{t}$ is given by

$$
q_{e i}=\left(\epsilon_{2}+\epsilon_{3}\right) \int_{\theta_{x i-1}}^{\theta_{2 i}}\left\{\sum_{n=0}^{\infty} a_{n} \cos \left(n+\frac{1}{2}\right) \theta\right\} d \theta .
$$

Eqs. (7.17) and (7.18) are closely related to conditions (7.5) and (7.6), respectively.

Problem (b) can be reduced to the following sine series equations:

$$
\begin{aligned}
& \sum_{n=1}^{\infty} a_{n} \sin n \theta=0, \quad \theta_{2 i-2}<\theta<\theta_{2 i-1}, \quad i=1, \cdots, N+1, \\
& \sum_{n=1}^{\infty} n^{-1}\left(1+H_{n}\right) a_{n} \sin n \theta=v_{i}, \quad \theta_{2 i-1}<\theta<\theta_{2 i}, \quad i=1, \cdots, N,
\end{aligned}
$$

where $\theta_{i}=\pi x_{i} / r$ and the charge $q_{o i}$ is given by

$$
q_{o i}=\left(\epsilon_{2}+\epsilon_{3}\right) \int_{\theta_{* i-2}}^{\theta, i}\left\{\sum_{n=1}^{\infty} a_{n} \sin n \theta\right\} d \theta .
$$

Eqs. (7.20) and (7.21) are related to conditions (7.5) and (7.6), respectively.

To show how the series equations method works, we give numerical results of some simple examples.

1. Chestnut's [1] example $(N=1)$. Parameters of the transmission line system are as follows:

$$
\begin{gathered}
\epsilon_{1}=\epsilon_{2}=\epsilon_{3}=\epsilon \\
h_{1}=1.0, h_{2}=0, h_{3}=1.0 \\
x_{1}=0.1, x_{2}=0.5, x_{3}=r=l(l=0.75,1.25) .
\end{gathered}
$$

Since $N=1$, series equations are reduced to a single integral equation. The integral equation (5.21) or (6.18) was approximately replaced by $m$ simultaneous linear equations and solved numerically. The series involving $H_{n}$ in the kernel of the integral equation was truncated at $n=n_{1}$ where $\left|H_{n_{1}}\right|<\left|H_{1}\right| \cdot 10^{-7}$. In Table 1 , we give the numerical 
TABLE 1.

Numerical results of Chestnut's [1] example (note: EXT in Chestnut's result denotes the value $C(m / 3, m / 5, m)$ extrapolated by his method).

\begin{tabular}{|c|c|}
\hline$l=0.75$ & $n_{1}=3$ \\
\hline$m$ & $C_{e}(m) / \epsilon$ \\
\hline 4 & 2.588624 \\
6 & 2.589841 \\
8 & 2.590163 \\
10 & 2.590281 \\
15 & 2.590367 \\
20 & 2.590386 \\
$C_{6}^{\text {EX T }}(4,6)$ & 2.590353 \\
$C_{\dot{E}}^{\text {EX T }}(6,8)$ & 2.590398 \\
Exact & 2.590398 \\
\hline
\end{tabular}

\begin{tabular}{|c|c|}
\hline$l=0.75$ & $n_{1}=3$ \\
\hline$m$ & $C_{0}(m) / \epsilon$ \\
\hline 4 & 4.236568 \\
6 & 4.373639 \\
8 & 4.415367 \\
10 & 4.427795 \\
15 & 4.432731 \\
20 & 4.432973 \\
$C_{0}^{\text {EXT }}(4,6,8)$ & 4.433630 \\
$C_{0}^{\text {EX T }}(6,8,10)$ & 4.433066 \\
Exact $^{\text {Exat }}$ & 4.433000 \\
\hline
\end{tabular}

\begin{tabular}{|c|c|}
\hline \multicolumn{2}{|c|}{ Chestnut's result [1] } \\
\hline 15 & 2.5984 \\
EXT & 2.5918 \\
\hline 30 & 2.5938 \\
EXT & 2.5914 \\
\hline
\end{tabular}

\begin{tabular}{|c|l|}
\hline \multicolumn{2}{|c|}{ Chestnut's result [1] } \\
\hline 15 & 4.4680 \\
EXT & 4.4483 \\
\hline 30 & 4.4435 \\
EXT & 4.4362 \\
\hline
\end{tabular}

\begin{tabular}{|c|c|}
\hline$l=1.25$ & $n_{1}=5$ \\
\hline$m$ & $C_{\bullet}(m) / \epsilon$ \\
\hline 4 & 1.971626 \\
6 & 1.972435 \\
8 & 1.972659 \\
10 & 1.972741 \\
15 & 1.972800 \\
20 & 1.972814 \\
$C_{e}^{\text {EXT }}(4,6)$ & 1.972775 \\
$C_{e}^{\text {EXT }}(6,8)$ & 1.972822 \\
Exact & 1.972822 \\
\hline
\end{tabular}

\begin{tabular}{|c|c|}
\hline$l=1.25$ & $n_{1}=5$ \\
\hline$m$ & $C_{0}(m) / \epsilon$ \\
\hline 4 & 3.965087 \\
6 & 3.999429 \\
8 & 4.004268 \\
10 & 4.005020 \\
15 & 4.005230 \\
20 & 4.005259 \\
$C_{0}^{\text {EXT }}(4,6,8)$ & 4.005062 \\
$C_{0}^{\text {EXT }}(6,8,10)$ & 4.005158 \\
Exact $^{\text {Exact }}$ & 4.005277 \\
\hline
\end{tabular}

\begin{tabular}{|c|c|}
\hline \hline \multicolumn{2}{|c|}{ Chestnut's result [1] } \\
\hline 15 & 1.9780 \\
EXT & 1.9746 \\
\hline 30 & 1.9745 \\
EXT & 1.9733 \\
\hline
\end{tabular}

\begin{tabular}{|c|c|}
\hline \hline \multicolumn{2}{|c|}{ Chestnut's result [1] } \\
\hline 15 & 4.0314 \\
EXT & 4.0147 \\
\hline 30 & 4.0142 \\
EXT & 4.0080 \\
\hline
\end{tabular}


TABLE 2.

Numerical results of capacitance matrix of the four-conductor transmission line system.

\begin{tabular}{|c|c|c|}
\hline$C_{i j} / \epsilon$ & Computed & $\begin{array}{c}\text { Results of } \\
\text { Kammler } \\
(r=\infty)\end{array}$ \\
\hline $\begin{aligned} C_{11} / \epsilon & =C_{44} / \epsilon \\
C_{22} / \epsilon & =C_{33} / \epsilon \\
C_{12} / \epsilon= & C_{21} / \epsilon=C_{34} / \epsilon=C_{43} / \epsilon \\
C_{23} / \epsilon & =C_{32} / \epsilon \\
C_{13} / \epsilon= & C_{31} / \epsilon=C_{24} / \epsilon=C_{42} / \epsilon \\
C_{14} / \epsilon & =C_{41} / \epsilon\end{aligned}$ & $\begin{array}{r}2.89139 \\
3.29377 \\
-1.00605 \\
-0.97670 \\
-0.07948 \\
-0.01247\end{array}$ & $\begin{array}{r}2.8914 \\
3.2938 \\
-1.0061 \\
-0.9767 \\
-0.0795 \\
-0.0125\end{array}$ \\
\hline
\end{tabular}

result achieved by the method presented in this paper and the exact value obtained by the conformal mapping method for $C_{0}$ and $C_{0}$, where $C_{e}=C_{11}+C_{12}, C_{0}=C_{11}-C_{12}$. The orders of the error in $C_{\text {。 }}$ and $C_{0}$ are $0\left(1 / \mathrm{m}^{\alpha}\right)$, where $\alpha \geqq 3$, while the method of a Fredholm integral equation of the first kind [1], [2] produce the error of the order of $\beta_{1} / m^{2}+\beta_{2} / m^{3}$, where $\beta_{i}$ is a certain constant. The extrapolation technique described by [Kammler [2] can be utilized for $C_{c}$ and $C_{0}$. In this case they are given by

$$
\begin{aligned}
C_{0}^{\mathrm{EXT}}\left(m_{1}, m_{2}\right) & =\frac{m_{2}^{3} C_{e}\left(m_{2}\right)-m_{1}^{3} C_{e}\left(m_{1}\right)}{m_{2}^{3}-m_{1}^{3}}, \\
C_{0}^{\mathrm{EXT}}\left(m_{1}, m_{2}, m_{3}\right) & =C_{0}\left(m_{3}\right)-\frac{\left\{C_{0}\left(m_{3}\right)-C_{0}\left(m_{2}\right)\right\}^{2}}{C_{0}\left(m_{1}\right)+C_{0}\left(m_{3}\right)-2 C_{0}\left(m_{2}\right)} \quad\left(m_{1}+m_{3}=2 m_{2}\right) .
\end{aligned}
$$

Extrapolated values are also given in Table 1. Since this example is very simple, the classical conformal mapping method can be applied. The method presented in this paper is feasible for practically important problems to which the conformal mapping method is useless, e.g. those of $\epsilon_{1} \neq \epsilon_{2} \neq \epsilon_{3}$.

2. Four-conductor system $(N=2)$. Parameters of the transmission line are as follows:

$$
\begin{gathered}
\epsilon_{1}=\epsilon_{2}=\epsilon_{3}=\epsilon, \\
h_{1}=0.5, h_{2}=0, h_{3}=0.5, \\
x_{1}=0.05, x_{2}=0.25, x_{3}=0.35, x_{4}=0.55, x_{5}=r=2.5 .
\end{gathered}
$$

This example problem is equivalent to that of Kammler [2] except $r=\infty$ in Kammler's example. Numerical results are given in Table 2. These computations were carried out using the computer system FACOM 230-60 at the Data Processing Center, Kyoto University.

\section{REFERENCES}

[1] P. C. Chestnut, On determining the capacitances of shielded multiconductor transmission lines, IEEE Trans. MTT-17, 734-745 (1969)

[2] D. W. Kammler, Calculation of characteristic admittances and coupling coefficients for strip transmission lines, IEEE Trans. MTT-16, 925-937 (1968) 
[3] T. Kiyono and M. Shimasaki, On the solution of Laplace's equation by certain dual series equations SIAM J. Appl. Math. 21, 245-257 (1971)

[4] J. S. Lowndes, Some triple series equations involving Jacobi polynomials, Proc. Edin. Math. Soc. 16, 101-108 (1968)

[5] B. Noble, Some dual series equations involving Jacobi polynomials, Proc. Camb. Phil. Soc. 59, 363-371 (1963)

[6] I. N. Sneddon, Mixed boundary value problems in potential theory, North-Holland, 1966

[7] R. P. Srivastav, Dual series relations. IV: dual relations involving series of Jacobi polynomials, Proc. Roy. Soc. Edin. A66, 185-191 (1964)

[8] K. N. Srivastava, On triple series equations involving series of Jacobi polynomials, Proc. Edin. Math. Soc. 15, 221-231 (1967) 